九州大学学術情報リポジトリ

Kyushu University Institutional Repository

\title{
STEM observation of tungsten tips sharpened by field-assisted oxygen etching
}

Onoda, Jo

Department of Molecular and Material Sciences, Kyushu University

Mizuno, Seigi

Department of Molecular and Material Sciences, Kyushu University

Ago, Hiroki

Institute for Materials Chemistry and Engineering, Kyushu University

http://hdl. handle. net/2324/26432

出版情報：Surface Science. 604 (13/14)，pp. 1094-1099，2010-07-15. Elsevier バージョン:

権利関係: (C) 2010 Elsevier B.V. 
STEM observation of tungsten tips sharpened by field-assisted oxygen etching

\author{
Jo Onoda $^{\text {a }}$, Seigi Mizuno ${ }^{\mathrm{a},{ }^{*}}$ and Hiroki Ago ${ }^{\mathrm{b}}$ \\ ${ }^{a}$ Department of Molecular and Material Sciences, Kyushu University, Kasuga, Fukuoka \\ 816-8580, Japan \\ ${ }^{\mathrm{b}}$ Institute for Materials Chemistry and Engineering, Kyushu University, Kasuga, \\ Fukuoka 816-8580, Japan
}

\begin{abstract}
Tungsten tips oriented toward the $<111>$ direction were fabricated by field-assisted $\mathrm{O}_{2}$ etching, and observed by means of scanning transmission electron microscopy (STEM) and field ion microscopy (FIM). The radii of curvature of the tip apexes were sharpened from 16-20 nm to less than $2 \mathrm{~nm}$. The $\mathrm{O}_{2}$ etching is considered to start from the $\mathrm{O}_{2}$ imaging region depending on the field distribution around the tip apex and shank. We estimated the effect of field distribution derived from a shank shape and applied bias voltage. The results showed that the tip with a cylindrical shank before the $\mathrm{O}_{2}$ etching became sharper than tips having an initial paraboloidal shape, with respective cone angles (defined in Fig. 3) of $58^{\circ}$ and $80^{\circ}$. The field emission (FE) patterns of these etched tips became a single spot derived from the W(111) plane, and their opening angles (defined by the full width at half maximum) were $14.4^{\circ}$ and $7.8^{\circ}$, respectively.
\end{abstract}

Keywords: Tungsten tips, Field emission (FE), Field ion microscopy (FIM), Scanning transmission electron microscopy (STEM)

* Corresponding author.

E-mail address: mizuno@mm.kyushu-u.ac.jp (S. Mizuno) 


\section{Introduction}

Field emissions (FEs) from atomically sharp tips have high brightness and spatial coherency. These properties make them of interest for use as point FE guns in lens-less applications, such as scanning Auger electron microscopy [1], projection microscopy [2], near-field emission scanning electron microscopy [3] and low-energy electron diffraction on the sub-micron order [4]. For these high-resolution electron-beam instruments, the apex structures of tips are crucial. Moreover, atomically well defined sharp tips are indispensable for spectroscopy and the measurement of conductivity in the field of scanning probe microscopy (SPM).

One type of ultra-sharp tip is widely known as a single-atom termination tip, which has three-sided pyramid terminated apex in the nanoscale [5-12]. Single-atom tips on which noble metals are deposited [9-12] are especially thermodynamically stable; even if the topmost atomic structures are destroyed, they can return to the single-atom termination tip only by heat treatments. On the other hand, Rezeq et al. reported another type of ultra-sharp tip, fabricated by field-assisted nitrogen etching [13]. They achieved atomically sharp tips by introducing nitrogen gas under field ion microscopy (FIM) conditions at liquid-nitrogen temperature and by controlling the applied bias voltage carefully. Rahman et al. succeeded in sharpening W tips by field-assisted oxygen etching [14] and evaluated the $\mathrm{FE}$ properties of $\mathrm{N}_{2}$ or $\mathrm{O}_{2}$ etched tips by a Fowler-Nordheim (FN) plot [15]. The physical phenomena behind these fabrications were studied by Rendulic et al. [16] and Cranstoun et al. [17] as 'field corrosion' as early as the late 1960s and early 1970s; however, the importance for various applications as those mentioned above has only recently been recognized.

The mechanism of field-assisted $\mathrm{N}_{2}$ etching can be explained as follows. Due to the gradient of electric potential of the FIM bias, nitrogen molecules cannot be adsorbed onto the apex where a high electric field exists, but can be adsorbed on the peripheral shank where the electric field is lower. Adsorbed nitrogen molecules form tungsten protrusions and then enhance the electric field locally. As a result, substrate tungsten atoms are evaporated by the field. As for field-assisted $\mathrm{O}_{2}$ etching, though the detailed mechanism is still unknown, desorption of tungsten atoms is supposed to occur around the peripheral shank as discussed in the results of FIM [14]. We discuss the mechanism of $\mathrm{O}_{2}$ etching later.

Although we can monitor the change of the tip apex by FIM, it is hard to know the shape of the peripheral region where the field-assisted $\mathrm{O}_{2}$ etching is proceeding. James and Valdre observed sharp electrochemically etched tips by scanning transmission 
electron microscopy (STEM) and compared their FE properties by FN plots [18]. Here, we report a direct observation of $\mathrm{O}_{2}$-etched $\mathrm{W}$ tips by STEM. The remarkable result was that the apex of very sharp tips could be seen by STEM, and those radii of curvatures were less than $2 \mathrm{~nm}$. The analysis of the electric field simulations around the tip apexes provided us an important idea of how the sharpness was developing.

\section{Experiment}

The tips used in this study were made from single-crystal $(0.25 \mathrm{~mm} \phi) \mathrm{W}<111>$ oriented wires and prepared by electrochemical etching in $2 \mathrm{M} \mathrm{NaOH}$ solution. They were welded on tantalum wires $(0.3 \mathrm{~mm} \phi)$ which were attached to tantalum rods (2 $\mathrm{mm} \phi)$. Tip holders were loaded into a FIM chamber with a base pressure of $4 \times 10^{-7} \mathrm{~Pa}$ and set in front of the FIM screen [combination of a two-stage microchannel plate (MCP) and a phosphor screen $(70 \mathrm{~mm} \phi)]$. When we carried out FIM observation and field-assisted $\mathrm{O}_{2}$ etching, $\mathrm{He}\left(1 \times 10^{-3} \mathrm{~Pa}\right)$ was used as an imaging gas at liquid- $\mathrm{N}_{2}$ temperature. The MCP was placed $70 \mathrm{~mm}$ away from the tip apex. FIM and field emission microscopy (FEM) images were monitored by an intensified charge-coupled device camera. An extractor (inner diameter $3 \mathrm{~mm}$ ) was grounded and set in front of the tips. We grounded the MCP input electrode and applied $1.3 \mathrm{kV}$ to the MCP output electrode when we observed FEM images. The threshold bias of FE is defined as the voltage at which we could observe FE patterns on the screen. The current of FE was too low to measure at the threshold bias, but it became $0.1 \mathrm{pA}$ when we increased the bias $20 \mathrm{~V}$ (negative on the tip) from its threshold value.

The tip apexes were cleaned by field evaporation. The cleaned tips were taken out of the FIM chamber and transferred to a STEM chamber (S-4800 FE-SEM: Hitachi High-Tech). As we discuss in a later chapter, a certain amount of carbon contamination at the apex is inevitable during STEM observation by high-energy primary electron beams. After STEM observations we restored the tips to the FIM chamber. The contaminations were removed by field evaporation, carefully increasing the positive bias in the FIM operation. We successfully fabricated very sharp tips by field-assisted $\mathrm{O}_{2}$ etching, the detailed procedures of which have already been reported [14]; then the tips were transferred to the STEM chamber again to observe the results of the $\mathrm{O}_{2}$ etching.

\section{Results and Discussion}




\subsection{STEM observation of tips fabricated by field-assisted $\mathrm{O}_{2}$ etching}

We fabricated four tips A - D and obtained their STEM images as shown in Figs. 1, 2. A STEM image of the tip A before field-assisted $\mathrm{O}_{2}$ etching is shown in Fig. 1(a). The shape of the tungsten tip was confirmed as a black shadow in the STEM image. The light gray shadow surrounding the tip was carbon contamination caused by the STEM operation at the high accelerating voltage of $30 \mathrm{kV}$ in the STEM chamber $\left(10^{-4} \sim 10^{-3}\right.$ $\mathrm{Pa}$ ). We could tell this from the fact that the thickness of the carbon contamination (light gray shadow) in the STEM image became thicker and thicker during the STEM operation while that of the tungsten tip (black shadow) did not change. Before the STEM observation, the tip apex was cleaned by field evaporation to be hemispherical in shape, and its best imaging voltage (BIV) in the FIM was $6.0 \mathrm{kV}$ as shown in Fig. 1(b). From the corresponding ball model represented in Fig. 1(c), the radius of curvature observed in the FIM image was estimated to be $18.3 \mathrm{~nm}$, which was in good agreement with that obtained from the STEM image. The FE pattern had four spots derived from the centered <111> direction and three equivalent <411> directions as shown in Fig. 1(d). The field-assisted $\mathrm{O}_{2}$ etching made the tip apex distinctively sharp as represented in the STEM image in Fig. 1(e). A conical shape of the tip apex was obtained. Only a few atoms were visible at the topmost (111) plane in the FIM image in Fig. 1(f). Its BIV became $1.7 \mathrm{kV}$ and the radius of curvature was estimated to be $1.8 \mathrm{~nm}$ from the ball model shown in Fig. 1(g). The BIV of the FIM decreased to be only one-third of the initial state, whilst the radius of curvature became one-tenth of the initial state after the $\mathrm{O}_{2}$ etching. The reason was that the field reduction factor increased after the etching [15] due to the change of the shape of the tip from cylindrical to conical. Therefore, it appears that a rather high voltage is necessary to achieve the BIV at the sharpened tip apex. The four spots of FE pattern in Fig. 1(d) changed into a single spot as shown in Fig. 1(h) by the etching. The threshold bias decreased from $-340 \mathrm{~V}$ to $-170 \mathrm{~V}$, which was only half of the initial state.

We tried the above procedures for the other three tips, B - D, and succeeded in every experiment. STEM images of the tips B - D are represented in Figs. 2(a)-(c). Corresponding STEM images after the $\mathrm{O}_{2}$ etchings are shown in Figs. 2(d)-(f). The sharpening ability of the $\mathrm{O}_{2}$ etching was again confirmed. Their radii of curvature were also decreased from a range of $16-20 \mathrm{~nm}$ to $1.8 \mathrm{~nm}$. BIVs of the FIM or threshold biases of FE were changed into one-third or one-half that of the initial states after the $\mathrm{O}_{2}$ etching. The results are summarized in Table I. 


\subsection{Mechanism of the $\mathrm{O}_{2}$ etching}

We supposed that oxygen atoms or molecules can adsorb at the $\mathrm{O}_{2}$ imaging region due to 'field-induced chemisorption' [19-21], and that tungsten oxides are then formed as described in former research. These tungsten oxides are shown as bright spots at the $\mathrm{O}_{2}$ imaging region (Fig. 5 of Ref. [14]). Since tungsten atoms in the oxides have lower binding energy than metal tungsten, they are field evaporated by local field or thermal fluctuations. Such field evaporation of tungsten oxides seems to occur successively at the $\mathrm{O}_{2}$ imaging region, especially at the higher field region in the $\mathrm{O}_{2}$ imaging belt. Cranstoun and Anderson also explained the mechanism of oxygen etching in Ref. [17] whereby oxygen molecules adsorbed on the lower field region form $\mathrm{W}_{\mathrm{n}} \mathrm{O}_{\mathrm{m}}$ molecules and then migrate from the shank toward the apex because of the chemical potential gradient. Once reaching the high field region, they get field evaporated. We suggest that the actual $\mathrm{O}_{2}$ etching is caused by one or both of the above processes.

As we described previously [14], explosion-like $\mathrm{O}_{2}$ etching was observed, and we explained that it was a massive field evaporation of tungsten oxide layers at the peripheral shank. However, this phenomenon is also explained by the local field evaporation of tungsten oxide. When the $\mathrm{O}_{2}$ etching region is far from the tip apex and the shape of the tip is very smooth, the $\mathrm{O}_{2}$ etching makes a dipped ring structure at the $\mathrm{O}_{2}$ etching region, but the tip apex of the tungsten remains and does not field evaporate at once because of the small fluctuation of the field strength. However, at some critical point, the field evaporation of large numbers of tungsten atoms above the dipped ring structure eventually occurs. This is observed as an explosion-like etching. On the other hand, when the $\mathrm{O}_{2}$ etching region is near the tip apex or the shape of the tip is rough, the $\mathrm{O}_{2}$ etching causes local field evaporations of the tungsten and the etching proceeds gradually. This explanation is consistent with the experimental finding that explosion-like etching was often observed at the initial stage while gradual etching was dominant at the final stage of the $\mathrm{O}_{2}$ etching. In the present study, tip D showed no explosion-like etching but was etched only gradually. In order to comprehend the origin of the explosion-like $\mathrm{O}_{2}$ etching, we have to observe the dipped ring shape directly by STEM and analyze the material of the field-evaporated mass by atom-probe FIM to determine whether it is tungsten oxide or pure tungsten.

\subsection{Sharpening process of the tips}

The STEM observation provided us a wide view of the tip shape; therefore, we were able to analyze the etched region in real space. In our former research on $\mathrm{O}_{2}$-etched tips [14], we observed the crystallographic orientation at the tip apex by FIM and confirmed 
that the $\mathrm{O}_{2}$-etched tip had a conical shape without any obvious faceting. Therefore, we suggested that the shape of the $\mathrm{O}_{2}$ etched tip only depends on the distribution of the electric field around the tip apex but not on how the gas molecules or atoms tend to adsorb on the crystal surface.

The shape of an etched tip depends on the bias control during the etching. At first, we etched tips to be as sharp as possible. The FIM bias voltage was adjusted at the He-BIV of the topmost tungsten atoms and was reduced with etching to keep prevent evaporation. If operation of the voltage is ideal, the shape of an etched tip will be conical with a cone angle $\theta_{\text {cone }}$ [defined as shown in Fig. 2(g)] corresponding with the starting point of the etching. We prepared two tips $\mathrm{B}$ and $\mathrm{C}$ whose radii of curvature were similar (18 and $16 \mathrm{~nm}$ ), but whose shank shapes were different (cylindrical and parabolidal) as shown in Figs. 2(a) and (b). In order to investigate the effect of the field distribution derived from initial shank shapes, we simulated the electric potential by ion optics simulation software (SIMION, Idaho National Engineering and Environmental Laboratory). Fig. 3 shows the electric potential near the tip apexes before the $\mathrm{O}_{2}$ etching. The left side represents the case of a cylindrical tip and the right shows the case of a paraboloidal one. We identified the field strength of $44 \mathrm{~V} / \mathrm{nm}$ at the apex for He best imaging, and calculated the field strength suitable for $\mathrm{O}_{2}$ imaging, $14.5 \mathrm{~V} / \mathrm{nm}$ [22]; these are marked with arrows in Fig. 3. The position of the $\mathrm{O}_{2}$ imaging belt on the shank in the case of the cylindrical tip was further from the tip apex than that in the case of the paraboloidal tip. We supposed that $\mathrm{O}_{2}$ imaging region was almost equal to the $\mathrm{O}_{2}$ etching region as described in the above section. Therefore, we expected that the cone angle that originated from a cylindrical shank would be narrower than that from a conical one if our bias operation was good. The actual bias control was not very good and many topmost tungsten atoms were evaporated during the $\mathrm{O}_{2}$ etching. However, the cone angles ultimately obtained showed a clear difference; tip B became $58^{\circ}$ while tip $\mathrm{C}$ became $80^{\circ}$ after the $\mathrm{O}_{2}$ etching as shown in Figs. 2(d) and (e). Such results were confirmed by several other tips. We verified the sharpening processes by observing FIM carefully; i.e., we counted the number of removed tungsten layers as accurately as possible and estimated the radii of curvature at several time points during the $\mathrm{O}_{2}$ etching. Figs. 4(a) and (b) represent the sharpening processes of tip B and tip C, respectively. We also estimated individual $\mathrm{O}_{2}$ imaging regions by SIMION and marked them with arrows numbered $1-3$. The $\mathrm{O}_{2}$ imaging regions of tip $\mathrm{B}$ were always further from the apex than those of tip $\mathrm{C}$ during etching. We conclude that a tip with a cylindrical shank tend to have a smaller cone angle after the $\mathrm{O}_{2}$ etching under the condition that the field strength at the apexes is high enough to allow He-gas imaging but controlled not to 
field-evaporate tungsten atoms.

Next, we investigated sharpening of a tip at a lower bias voltage, at which He-ionized spots at the center of the FIM image could not be seen. In that case, the starting position of adsorption is supposed to approach the tip apex. Therefore, the $\mathrm{O}_{2}$ etching starts near the tip apex and the cone angle will be larger. We tried using tip D, which had a cylindrical shank as shown in Fig. 2(c). As a result, the cone angles of tip D after the $\mathrm{O}_{2}$ etching became $80^{\circ}$, which was larger than that of the tip B, $58^{\circ}$.

The experimental results for tips $\mathrm{B}$ - D showed that the sharpening process of the $\mathrm{O}_{2}$ etching depends on the field distribution around the tip; the etched tip shape depends on the initial tip shape and how we apply bias voltage to the tip. This means that we can control the cone angle to some extent by controlling the applied bias voltage.

Indeed, we expected that the massive field evaporation might have influenced the cone angles. STEM images and the obtained cone angles, however, did not show us a critical difference between the explosion-like and the gradual $\mathrm{O}_{2}$ etchings. Although they may contribute partially to the change of the tip shank, it is hard to distinguish the influence of the explosion-like $\mathrm{O}_{2}$ etching to the tip shapes from STEM images at the initial and etched states alone. We must research STEM images at the middle of the $\mathrm{O}_{2}$ etching sharpening process in greater detail.

\subsection{Characteristics of the $\mathrm{O}_{2}$-etched tips}

FEM images of tip B and tip D before the $\mathrm{O}_{2}$ etching are represented in Figs. 5(a) and (b), respectively. We obtained them at similar bias voltages $(-310 \mathrm{~V}$ and $-320 \mathrm{~V}$ respectively). After the $\mathrm{O}_{2}$ etching, bias voltages of $\mathrm{FE}$ decreased to $-150 \mathrm{~V}$ and $-240 \mathrm{~V}$, respectively, and the FE patterns changed to single spots as shown in Figs. 5(c) and (d). If one succeeds in sharpening a $<111>$-oriented $\mathrm{W}$ tip by $\mathrm{O}_{2}$ etching, only the (111) plane remains on the tip apex. The conical shape of the tip apex permits all of the FE to come from only the (111) plane. In other words, the FE is collimated to the <111> direction. Similar angular confinements were reported in previous studies of single-atom tips; their opening angles were in the range of $3-7^{\circ}$, and under some conditions less than $2^{\circ}[10,12]$. We also estimated the opening angle which was defined by the full width at half maximum (FWHM) after the $\mathrm{O}_{2}$ etching. Those of tip B and tip $\mathrm{D}$ were $14.4^{\circ}$ and $6.2^{\circ}$, respectively. The difference of opening angles is attributed to their cone angles; in other words, the wider the cone angle becomes, the narrower the opening angle becomes. This is due to the collimating ability of the conical shank at the vicinity of the apex. Cone angles also influenced the threshold bias voltage of FE; i.e., 
the smaller the cone angles were, the lower the threshold bias voltage became. The opening angles $\theta_{\text {open }}$ of the $\mathrm{O}_{2}$-etched tips A - D are summarized in Table. I. From these results, if a field emitter with a narrow opening angle is desired, only the shank near the tip apex should be etched. We also measured currents of FE from $\mathrm{O}_{2}$-etched tips and estimated the brightness. Tips with large cone angles like tip $\mathrm{C}$ and tip D emitted a current of $130 \mathrm{pA}$ stably at around $-340 \mathrm{~V}$ with opening angles of $8^{\circ}$. Their brightness reached $1.8 \times 10^{7} \mathrm{~A} \mathrm{~m}^{-2} \mathrm{sr}^{-1} \mathrm{~V}^{-1}$. On the other hand, tips with small cone angles like tip A and tip B emitted a current of 100 pA stably around at $-260 \mathrm{~V}$ with opening angles less than $16^{\circ}$. Therefore, we found that their brightness reached at least $4.8 \times 10^{6} \mathrm{~A} \mathrm{~m}^{-2} \mathrm{sr}^{-1}$ $\mathrm{V}^{-1}$. Tips with narrower cone angles have higher aspect ratios; therefore, they are suitable for SPM tips. They may produce good SPM images even if one scans the surfaces with rough terraces or deep steps. To obtain this kind of tip, we have to prepare the cylindrical tips in advance, but it is hard to control the shape of the shank at the sub-micron order during the stage of electrochemical etching. Nevertheless, an atomically sharp tip fabricated by the $\mathrm{O}_{2}$ etching will show good ability in SPM operation.

\section{Conclusion}

The shapes of tips fabricated by field-assisted $\mathrm{O}_{2}$ etching were observed using STEM. Their obtained radii of curvature were less than $2 \mathrm{~nm}$, corresponding with those obtained from FIM images. We concluded that the $\mathrm{O}_{2}$ etching occurred at the $\mathrm{O}_{2}$ imaging region. Electric distribution at the tip apex and shank is essential to the starting position of the $\mathrm{O}_{2}$ etching and the resultant shape of the tips. This means that the shape of the $\mathrm{O}_{2}$-etched tip is strongly dependent on what the initial tip shape is or how we apply the bias voltage to the tip. The detailed mechanism of the $\mathrm{O}_{2}$ etching was also discussed. Lastly, we successfully confirmed that the opening angles of the FE from the $\mathrm{O}_{2}$-etched tips were smaller if their cone angles were large.

Acknowledgements

This work was supported by KAKENHI (20340077) from the Ministry of Education, Culture, Sports, Science and Technology of Japan and the Industrial Technology Research Grant Program in '05 from NEDO of Japan. 
[1] B. Reihl, J.K. Gimzewski, Surf. Sci. 189-190 (1987) 36.

[2] H.-W. Fink, W. Stocker, H. Schmid, Phys. Rev. Lett. 65 (1990) 1204.

[3] T. L. Kirk, L. G. De Pietro, D. Pescia, U. Ramsperger, Ultramicroscopy 463-466 (2009) 109.

[4] S. Mizuno, F. Rahman, M. Iwanaga, Jpn. J. Appl. Phys. 45 (2006) L178.

[5] H.-W. Fink, IBM. J. Res. Dev. 30 (1986) 460.

[6] H.-W. Fink, Phys. Scr. 38 (1988) 260.

[7] V. T. Binh, N. Garcia, J. Phys. I (France) 1 (1991) 605.

[8] V. T. Binh, N. Garcia, Ultramicroscopy $42-44$ (1992) 80.

[9] K. Nagaoka, H. Fujii, K. Matsuda, M. Komaki, Y. Murata, C. Oshima, T. Sakurai, Appl. Surf. Sci. 182 (2001) 12.

[10] H.-S. Kuo, I.-S. Hwang, T.-Y. Fu, J.-Y. Wu, C.-C. Chang, T.T. Tsong, Nano Lett. 4 (2004) 2379.

[11] E. Rokuta, T. Itagaki, D. Miura, T. Moriyama, T. Ishikawa, B.-L. Cho, T.Y. Fu, T.T. Tsong, C. Oshima, Appl. Surf. Sci. 251 (2005) 205.

[12] E. Rokuta, H.-S. Kuo, T. Itagaki, K. Nomura, T. Ishikawa, B.-L. Cho, I.-S. Hwang, T.T. Tsong, C. Oshima, Surf. Sci. 602 (2008) 2508.

[13] M. Rezeq, J. Pitters, R. Wolkow, J. Chem. Phys. 124 (2006) 204716.

[14] F. Rahman, J. Onoda, K. Imaizumi, S. Mizuno, Surf. Sci. 602 (2008) 2128.

[15] J. Onoda, F. Rahman, S. Mizuno, e-J. Surf. Sci. Nanotech. 6 (2008) 152.

[16] K. D. Rendulic, Z. Knor, Surf. Sci. 7 (1967) 205.

[17] G. K. L. Cranstoun, J. S. Anderson, Surf. Sci. 35 (1973) 319.

[18] E. M. James, U. Valdrè, Inst. Phys. Conf. Ser. 147 (1995) 293.

[19] R. G. Fobes, H. J. Kreuzer, R. L. C. Wang, Appl. Sur. Sci, 94-95 (1996) 60.

[20] Y. D. Osovskii, Yu. G. Ptushinskii, V. G. Sukretnyi, B. A. Chuikov, V. K. Medvedev, Yu. Suchorski, Surf. Sci. 377-379 (1997) 664.

[21] Yu. Suchorski, Ultramicroscopy. 73 (1998) 139.

[22] E. W. Müller, T. T. Tsong, Field Ion Microscopy: Principles and Applications, American Elsevier Publishing Company, Inc. (1969). 
Fig. 1: STEM images, FIM images, corresponding ball models and FEM images of tip A before field-assisted $\mathrm{O}_{2}$ etching (a)-(d) and after field-assisted $\mathrm{O}_{2}$ etching (e)-(h). The black deficit shown in (h) is a defect of MCP.

Fig. 2: (a)-(c) STEM images of tips B-D before field-assisted $\mathrm{O}_{2}$ etching. (d)-(f) STEM images of tips B-D after field-assisted $\mathrm{O}_{2}$ etching. (g) Illustration of cone angle $\theta_{\text {cone }}$ and radius of curvature $R$ of tip $\mathrm{B}$ after field-assisted $\mathrm{O}_{2}$ etching [Figs. $2(d)]$.

Fig. 3: Simulations of electric equipotential lines near cylindrical (tip B) (a) and paraboloidal (tip C) (b) tips. Arrows indicate the $\mathrm{O}_{2}$-ionization regions.

Fig. 4: Schematic illustrations of the $\mathrm{O}_{2}$-etching sharpening processes for cylindrical (tip B; a) and paraboloidal (tip $\mathrm{C}$; b) tips. The $\mathrm{O}_{2}$-ionization region changed from the site indicated by arrows numbered 1 to the site numbered 3 .

Fig. 5: (a) and (b) FEM images of tip B and tip D before field-assisted $\mathrm{O}_{2}$ etching. (c) and (d) FEM images of tip $\mathrm{B}$ and tip $\mathrm{D}$ after field-assisted $\mathrm{O}_{2}$ etching. The black deficit shown in (c) is a defect of MCP.

Table I. Radii of curvature, BIVs and threshold FE voltages of tips A-D before and after field-assisted $\mathrm{O}_{2}$ etching. Cone and opening angles of tips A-D after field-assisted $\mathrm{O}_{2}$ etching are also represented. 

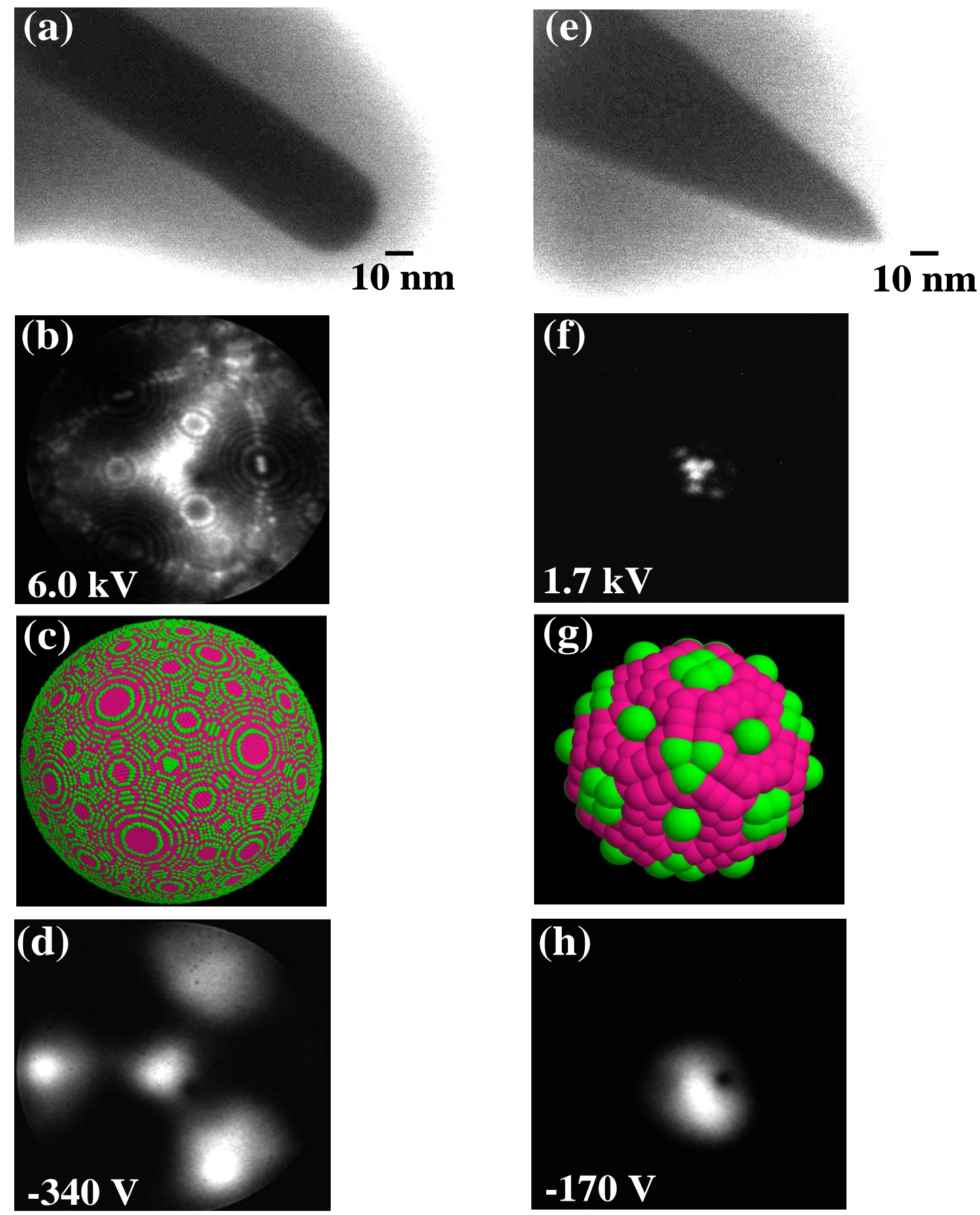

Fig. 1 


\section{(a)}

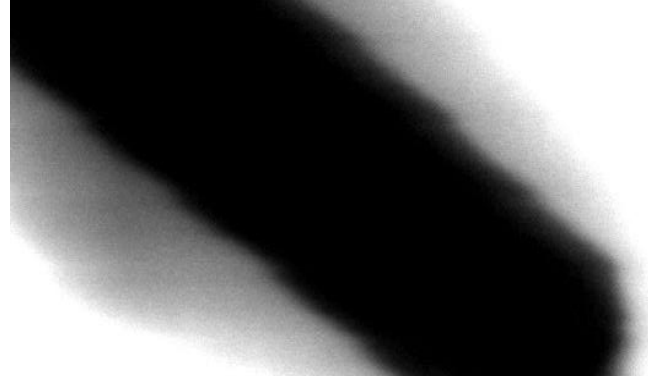

$1 \overline{\mathrm{nm}}$

(b)

$1 \overline{\mathrm{nm}}$

(c)

$10 \overline{\mathrm{nm}}$

(g)

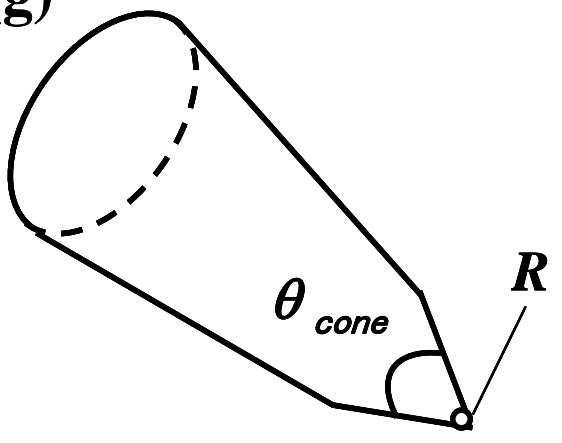

(d)

$1 \overline{\mathrm{nm}}$

(e)

$1 \overline{n m}$

(f)

$\overline{10} \mathrm{~nm}$

Fig. 2 


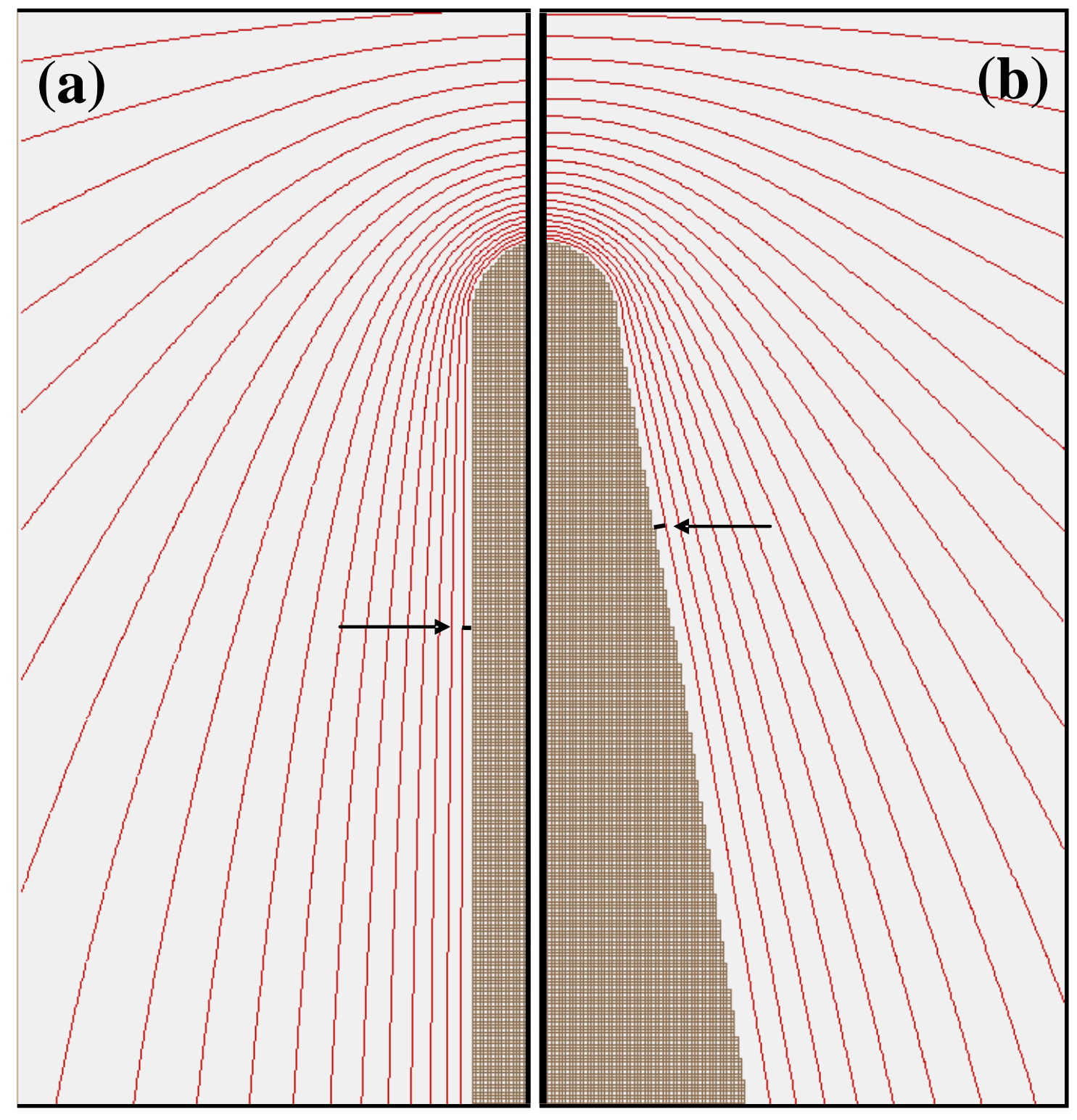

Fig. 3 

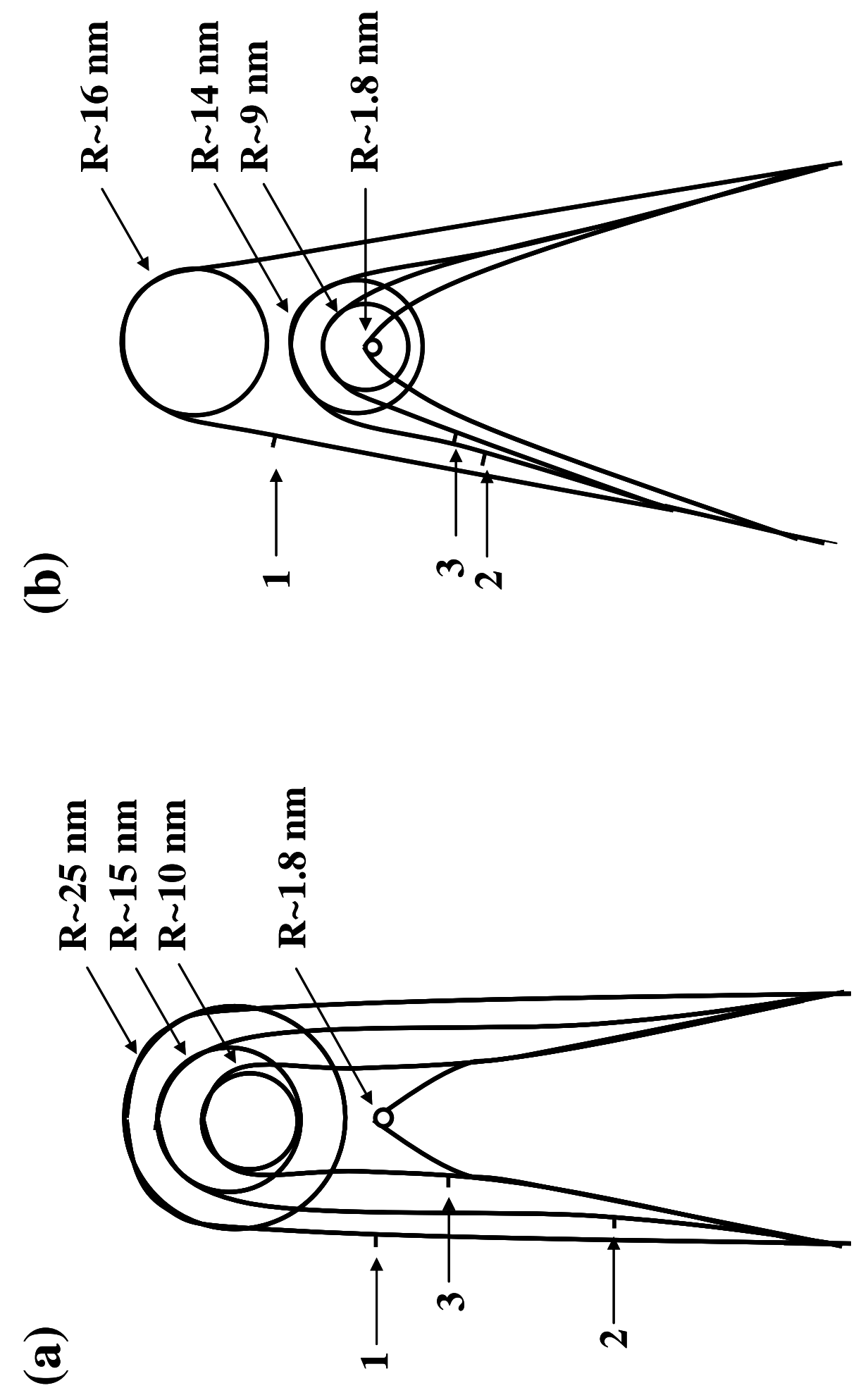

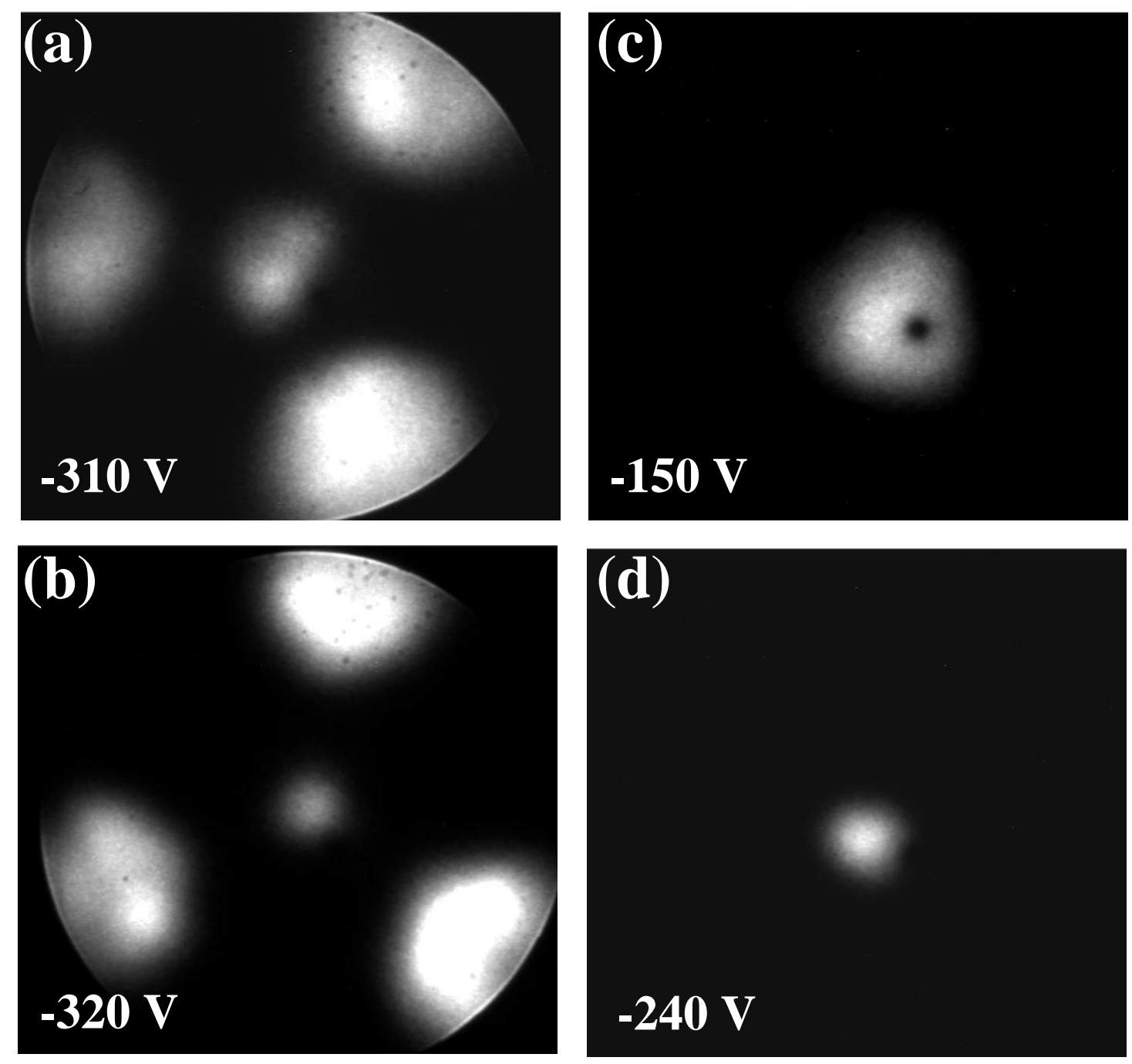

Fig. 5 


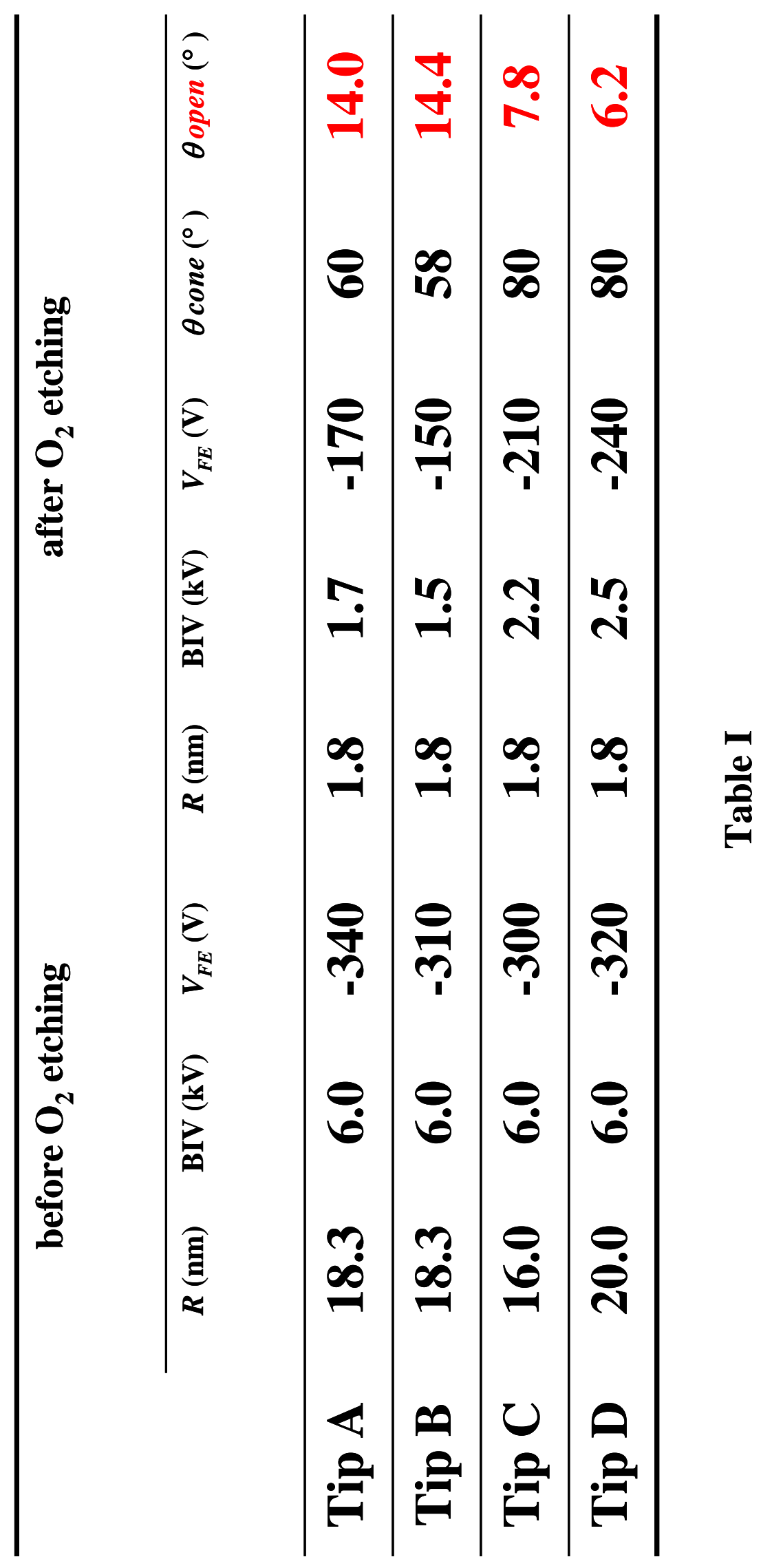

